\title{
Stressing Experience and Stigma of Covid-19 Pandemic: Analysis Of Pre-Service Nurses Intention Toward Infectious Diseases Centres in Ghana
}

\author{
Hannah Yawson, PhD Candidate \\ Principal, Twifo Praso Nurses \& Midwife Training College \\ Email: ber976@gmail.com
}

\begin{abstract}
This paper explored perceived stressing experience and perceived stigma of Coronavirus (COVID-19) pandemic among pre-service nurses and how these perceptual factors could affect their intention to work at infectious treatment centres in the future. The paper followed mixed analytical procedure where both quantitative and qualitative approaches were employed. Concurrent analytical procedures were adopted where both qualitative and quantitative data were collected concurrent through the use of questionnaire. Questionnaire and interview guide were used for the collecting the data. Multi-stage sampling technique was employed where cluster, stratified and simple random sampling techniques were employed concurrently. Sample size of 150 was used. However, 117 questionnaires were obtained and processed. 11 students were also interviewed. Content and thematic analysis was used to analyse the qualitative data while mean scale and standard multiple regression were employed for the quantitative analysis. The study revealed high perception score of stigmatisation, perceived high stressing experience, and high work-family conflict. The study further found that all the perceptual factors: perceived Covid-19 stigma, perceived covid-19 stressing experience and perceived covid-19 related work-family conflict have significant negative influence on the pre-service nurses' intention to provide care for infectious diseases. It is recommended that training of these pre-service nurses should emphasis these experiences and possible stigmas to minimise elements of surprise. Health administrators should regular gather data on nurses' experience and perceive stigmas of epidemics and infectious diseases and integrate them in their training programmes. There is no funding to report.
\end{abstract}

Keywords: Coronavirus (Covid-19) Pandemic, Pre-Service Nurses, Perceived Stressing Experience, Stigma, Work-Family Conflict and Pre-Service Nurses Intention

DOI: $10.7176 / \mathrm{JMPB} / 72-03$

Publication date: February $28^{\text {th }} 2022$

\section{Introduction}

It is often said, 'Healthy People, Healthy Nation'. In view of this, quality healthcare is a priority for all nations. However the recent respiration infection, Coronavirus (COVID-19) Pandemic has exposed weaknesses in the healthcare system globally. Covid-19 has not only brought most of the healthcare systems on their knees but also challenged the welfare of the healthcare practitioners and uncovered rather hidden health risk exposure of the healthcare providers. Prior to the current healthcare crisis, it is mostly those who are closely related to the healthcare system really know and understand the risk.

The World Health Organisation (WHO) reported as at August 2020 the estimated confirmed case to be $17,859,763$ and 685,179 related deaths occurred globally (WHO, 2020a). Statistics indicate that more than $10 \%$ of these global cases were among healthcare practitioners (WHO, 2020a). Healthcare practitioners in Ghana have also experienced and continued to be exposed to this risk. For instant, Ghana has lost six experienced healthcare practitioners and 2,000 affected healthcare workers as at July 2020. Among these healthcare practitioners, nurses are one of the highly exposed categories as they are usually the first point of contact to the infection in health facilities.

Notwithstanding, being occupationally exposed to a higher risk of infection, these healthcare practitioners provoke social stigma and continue to adapt to rigid work schedules. According to Andoh and Lu, Chen, Liu \& Zhu (2020), stigmatisation is the act of treating a person or an identifiable group of people, place, or country differently in an unfavorable manner because they had a health conditions or were associated with something people did not want. It is also referred to discontentment of or negative attitudes toward persons with certain diseases or characteristics that distinguish these people from other members of institution or society. In relation to the pandemic, Mayo Clinic, (2020) described COVID-19 related stigma as the disapproving or negative selfattitude, stemming from being infected with, or having close contacts with COVID-19, that leads to a spoiled identity. The Covid-19 stigma is not surprising though as researchers have revealed that stigmatisation is almost always associated with outbreak of any major pandemic or epidemic (Earnshaw, 2020; Morens \& Fauci, 2013; 
WHO, 2020b). Examples of such pandemics and epidemics in history are Ebola, Middle East Respiratory Syndrome (MERS), Human Immunodeficiency Virus (HIV), Hansen's disease, and currently the Coronavirus (COVID-19) which has also sparked stigma against infected persons despite their prestige.

In fact, the recent global pandemic COVID-19 has resulted in an untold degree of stigmatisation, distressing working condition at treatment centres, infectious risk and adapting to rigid work schedules (Duan, Bu \& Chen, 2020; Earnshaw, 2020; Xiao, 2020). Recent findings on studies have affirmed this. A study conducted by Do Duy, Nong, Van, Thu, Do Thu and Quang (2020) revealed in Vietnam 18\% healthcare workers felt unsafe to work in healthcare facilities after they went through the quarantine procedure; $10 \%$ felt social isolation and blameworthy by family members or relatives and friends and 34\% avoided contacts with neighbours or others in the community due to feeling stigmatised. It is not surprising that Xiao (2020) postulates that the COVID-19 has not only increased mortality rate but has also witnessed a considerable increase in psychological and mental health disorders. The Ministry of Health and Family Welfare in India also revealed that since the pandemic (COVID-19) is new its emergence and the rate at which it spread creates anxiety, tension, panic and confusion among the general public as a result bleed social stigma (Ministry of Health and Fa, 2020).

This social stigma goes beyond only a person who has contracted or is associated with infected persons. In these incidents, healthcare practitioners faced social isolation, public insults or harassment, refusal of public transportation use, and house eviction because of direct exposure to Covid-19 Patients (International Federation, 2020). Nevertheless, efforts put in place to fight the pandemic would be fruitless if health care practitioners are being stigmatised because studies have revealed that people who are stigmatised due to an epidemic or pandemic have to constantly battle with mental health disorders and psychological stress which are not good mediator for services provider (Bao et al., 2020; Rajkumar,2020; WHO, 2020b).

Besides, the direct consequences on the healthcare providers, it could trigger anxiety and negative perceptions among 'would be healthcare providers'. The waves of reported mistreatment against healthcare providers especial nurses, reported unpreparedness and stressing working, high covid-19 related work-family conflict and stigma (Duan et al, 2020; Earnshaw, 2020; Do Duy et al, 2020; International Federation, 2020; Rajkumar,2020; Xiao, 2020) could have implications on pre-service nurses intention in working at infectious diseases treatment centre in the future. Thus, although these pre-service nurses have not directly worked at Covid-19 treatment centres and may not even worked at these centres, their perceptual errors about stressing experience, covid-19 related work-family conflict and stigma could undermine their good intention as potential healthcare practitioners ready to accept assignments at any centre including infectious diseases treatment centres. It is therefore not only useful in theory but also for policy and practitioners to assess these pre-service nurses' opinion and perception of these negative waves surrounding nurses who are working at Covid-19 treatment centre and how these perceptual factors could affect their intentions to work at similar centres in the future. Despite these sound bases for extending the literature on Covid-19 dynamics to pre-service nurses, there is virtually no study in this respect. It is against these that this paper seeks to contribute to Covid-19 literature by exploring the perceived stressing experience and perceived stigma of Coronavirus (COVID-19) pandemic among pre-service nurses and how these perceptual factors could affect their intention to work at infectious treatment centres in the future.

\section{Literature Review}

Recent studies on COVID-19 pandemic have revealed distressing concerns in respect of stigmatisation, psychological stress, deepening work-family conflicts, rigid work schedules and infectious risk related factors. Outbreaks of recognised contagious illnesses like COVID-19 highlight the infectious risk of healthcare practitioners since they have direct exposure to Covid-19 Patients. Obviously, modes of transmission of the COVID-19 pandemic led to healthcare practitioners fear and anxiety. Chen, Lai and Tsay (2020) studied the nursing perspectives on the impacts of COVID-19 in China and discovered that nurses are key members of healthcare teams providing direct care to individuals. This made them more prone to contracting the infectious diseases. The study revealed psychological distress and social burdens experienced by healthcare professionals and nurses during this outbreak which could have implications on the interest and attitude of current and potential nurses toward availing themselves to contribute openly to support the fight against future outbreaks of infectious diseases.

Another study was conducted by Mostafa, Sabry and Mostafa (2020) on the COVID-19-related stigmatisation among a sample of healthcare workers in Egyptian. Mostafa et al (2020) found high COVID-19-related stigma score, personalised stigma, disclosure concern, negative self-image and concern with public attitudes about HCWs. The study further revealed that only $14.7 \%$ of all participants in the research received psychological 
support to cope with the COVID-19 pandemic. These distressing conditions, ill-treatment and sigma could affect the resolve of future healthcare practitioners including pre-service nurses and their intention to respond positively to outbreak of infectious diseases. Thus, it is critical to have baseline empirical evidence about these pre-service nurses so as to develop robust strategy for future response to outbreak. In China, Yan-Jie , Qing-E, Ling, Teris, Todd, Guo-Qing and Yu-Tao 2020) also reported similar findings.

In the Ghanaian literature, Adom, Mensah and Osei (2021) conducted a study on understanding COVID-19 related stigma and related psychological distress. The study focused on COVID-19 related stigma and discrimination against healthcare workers, COVID-19 recovered patients, suspected persons of COVID-19, Asians, and persons with travel history from COVID-19 hotspot countries. The results revealed that COVID-19 victims have faced various forms of stigma such as stereotyping, social exclusion, mockery, finger-pointing, and insults. It was concluded that most of the COVID-19 National Response Team in Ghana must put in place a robust psychosocial intervention plan for stigmatized persons to help them cope with the stigma and help in its prevention.

A critical review of the literature shows that many of the studies have focused on Covid-19 effect on direct victims such as healthcare workers, COVID-19 recovered patients, and suspected persons of COVID-19. However, besides the direct consequences on the healthcare providers, it could trigger anxiety and negative perceptions among 'would be healthcare providers'. The waves of reported mistreatment against healthcare providers especial nurses, reported unpreparedness and stressing working, high covid-19 related work-family conflict and stigma (Duan et al, 2020; Earnshaw, 2020; Do Duy et al, 2020; International Federation, 2020; Rajkumar,2020; Xiao, 2020) could have implications on pre-service nurses intention in working at infectious diseases treatment centre in the future. Thus, although these pre-service nurses have not directly worked at Covid-19 treatment centres and may not even worked at these centres, their perceptual errors about stressing experience, covid-19 related work-family conflict and stigma could undermine their good intention as potential healthcare practitioners ready to accept assignments at any centre including infectious diseases treatment centres. It is therefore not only useful in theory but also for policy and practitioners to assess these pre-service nurses' opinion and perception of these negative waves surrounding nurses who are working at Covid-19 treatment centre and how these perceptual factors could affect their intentions to work at similar centres in the future. Despite these sound bases for extending the literature on Covid-19 dynamics to pre-service nurses, there is virtually no study in this respect.

\section{Analytical Procedures}

The study follows mixed analytical procedures with survey design. Mixed approach incorporates elements of both qualitative and quantitative approaches in a same research for the broad purposes of span and depth of corroboration and understanding (Wisdom \& Creswell, 2013). The paper adopted the mixed approach because both numerical and non-numerical data are required for the creating of wider understanding of the topic under studied. Survey design involves the use of standardised questionnaires to produces in-depth data to establish baselines against which future comparisons can be made or analysed. Survey design is appropriate as the paper sought to explore data which are normally not unobservable such as pre-service nurses' opinion and perceptions about Covid-19 related dynamics (Fraenkel \& Wallen, 2001). Concurrent analytical procedures were adopted where both qualitative and quantitative data were collected concurrently. Questionnaire was used for the quantitative data while interview guide was also used collect the non-numerical or text data.

The target population is the pre-service Nurses Training Colleges of Central and Western Regions. Sample size of 150 was used. However, 117 questionnaires were obtained and processed. A multi-stage sampling technique was employed where cluster, stratified and simple random techniques were used. The study first used cluster to group the schools according to geographical locations, followed by stratified sampling to classify the students into three strata: First Years, Second Years and Third Years. Finally, the sample size of 150 was allocated to the strata as follows: $40 \%$ for third years and 30\% each for second and first years. More allocation was given to third years as they would soon enter the field and they are more likely to witness firsthand experience of Covid-19 treatment and exposure. Simple random sampling was applied to select the individual students. 117 out of the 150 questionnaires were received and processed. This represented $78 \%$ response rate. This is relatively high. 11 students were interviewed through the interview guide. Content and thematic analysis was used to analyse the qualitative data while mean scale and standard multiple regression were employed for the quantitative analysis

\section{Empirical Results}

The paper presents and discusses the empirical results in this section. The descriptive statistics are captured in Table 1. It can be seen from Table I that the mean age of the respondents is about 21-25 years. This indicates that 
the pre-service nurses who participated in the study are youthful who are at their prime age of their career. Thus, any evidence from data provided from these students could have long term implications. About $82.90 \%$ of the respondents fall within this category. 87.18 per cent of the respondents are single with 52.99 per cent of them are female. In terms of levels, 40.17 per cent of the participants are at level 300, 29.92 percent at level 100 and 29.91 percent at level 300 .

Table 1: Demographic Factors of Respondents

\begin{tabular}{llll}
\hline Variables & Freq. & $\mathbf{\%}$ & Obs \\
\hline Age & & & 117 \\
$<20$ Years & 18 & $15.38 \%$ & \\
21-25 Years & 97 & $82.90 \%$ & \\
26-30 Years & 2 & $1.72 \%$ & \\
>30 Years & 0 & $0 \%$ & \\
Marital Status & & & 117 \\
Single & 102 & $87.18 \%$ & \\
Married & 7 & $5.98 \%$ & \\
Divorced & 0 & $0 \%$ & \\
Widowed & 0 & $0 \%$ & \\
Co-Habitation & 8 & $6.84 \%$ & \\
Gender & & & 117 \\
Female & 62 & $52.99 \%$ & \\
Male & 55 & $47.01 \%$ & \\
Levels & & & 117 \\
First Year & 35 & $29.92 \%$ & \\
Second Year & 35 & $29.91 \%$ & \\
Third Year & 47 & $40.17 \%$ & \\
\hline
\end{tabular}

Source: Field survey, Yawson (2021)

\section{Reliability and Internal Consistency}

The reliabilities of the key variables of interest are reported in Table 2. The three main independent variables: perceived Covid-19 stressing experience (PCSE), perceptual Covid-19 stigma (PCS) and perceived work-family conflict of Covid-19 treatment (PCWFC) have Cronbach's alphas values of $0.773,0.826$ and 0.731 respectively. The dependent variable: Intention to Work at Infectious Treatment Centres (IWITC) has Cronbach's alpha value of 0.698 . This means that all the variables have relatively high inter-item reliability as all the alpha values are above the recommended threshold level of 0.60 (Meyers et al., 2013). This also implies relatively high convergent validity. It can therefore be concluded that there is high internal consistency of the data.

Analysis of the Extent of Perceived Covid-19 Stressing Experience (PCSE), Perceptual Covid-19 Stigma (PCS), Perceived Work-Family Conflict of Covid-19 Treatment (PCWFC) and Intention to Work at Infectious Treatment Centres (IWITC)

The study independently assessed pre-service nurses' perceptual factors and intention to work at infectious treatment centre. Table 3 reports the results. It can be seen from Table 3 that the pre-service nurses' perceived covid-19 stressing experience, perceived covid-19 stigma against nurses at treatment centres, perceived covid-19 related work-family conficts and the intention toward working at infectious treatment centre are very high as evident in the high mean scores. At the individual scale level, all the mean scores crossed the midpoint of 2.99 to the 'HIGH LEVEL REGION'.

The pre-service nurses (respondents) perceived that working at Covid-19 treatment centre would be distressing experience. This is denoted by mean score of 3.230 which is greater than the midpoint or cut-off point of 2.9 (Dess, Lumpkin \& McFarlin, 2005; Yeboah, 2011). The standard deviation is relatively low. The coefficient of variation $(\mathrm{CoV})$ of 0.068 suggests very low level of dispersion. Thus, about $93 \%$ of the pre-service nurses perceive the environment as quite distressing. This implies that although the pre-service nurses have not actually experienced working at Covid-19 treatment centre, they still have high negative perception about the environment.

Similarly, the perceived covid-19 stigma against nurses working at Covid-19 treatment centres is very high. This is ranked the highest among all the perceptual factors of pre-service nurses. The coefficient of variation of 0.032 
suggests almost all the pre-service nurses have this perception. Thus, about $96 \%$ of the pre-service nurses perceive this stigmatisation. Surprisingly, although most of the pre-service nurses in this study are not married (See Table 1), they still perceived that covid-19 has broaden the work-family conflict with about $89 \%$ of the respondents having same perception.

Table 2: Reliability and Internal Consistency

\begin{tabular}{|c|c|c|}
\hline Variables & Item No. & Cronbach Alpha Value \\
\hline \multirow[t]{8}{*}{ PCSE } & PCSE1 & \\
\hline & PCSE2 & \\
\hline & PCSE3 & \\
\hline & PCSE4 & \\
\hline & PCSE5 & \\
\hline & PCSE6 & \\
\hline & PCSE7 & \\
\hline & PCSE8 & 0.773 \\
\hline \multirow[t]{7}{*}{ PCS } & PCS1 & \\
\hline & PCS2 & \\
\hline & PCS3 & \\
\hline & PCS4 & \\
\hline & PCS5 & \\
\hline & PCS6 & \\
\hline & PCS7 & 0.826 \\
\hline \multirow[t]{6}{*}{ PCWFC } & PCWFC1 & \\
\hline & PCWFC2 & \\
\hline & PCWFC3 & \\
\hline & PCWFC4 & \\
\hline & PCWFC5 & \\
\hline & PCWFC6 & 0.731 \\
\hline \multirow[t]{7}{*}{ IWITC } & IWITC1 & \\
\hline & IWITC 2 & \\
\hline & IWITC 3 & \\
\hline & IWITC 4 & \\
\hline & IWITC 5 & \\
\hline & IWITC 6 & \\
\hline & IWITC 7 & 0.698 \\
\hline
\end{tabular}

Source: Field survey, Yawson (2021)

These high negative perceptual scores are consistent with some of the earlier findings that the waves of reported mistreatment against healthcare providers especial nurses, reported unpreparedness and stressing working, high covid-19 related work-family conflict and stigma (Duan et al, 2020; Earnshaw, 2020; Do Duy et al, 2020; International Federation, 2020; Rajkumar,2020; Xiao, 2020) could have implications on pre-service nurses intention in working at infectious diseases treatment centre in the future

Nevertheless, when the pre-service nurses were interrogated through the Intention to Work at Infectious Treatment Centres (IWITC) scale, it was revealed that the pre-service nurses have positive intention to work in the future at infectious treatment centre. This is exhibited by mean score of 3.789 which is higher than the cut-off mean of 2.9. The coefficient of variation stands at 0.025 indicating that about $97 \%$ of the pre-service nurses are ready to work at infectious treatment centre in the future. This is good news for the practice. However, it is important to acknowledge that all the assessments were conducted in isolation. Therefore, the conditional effect of the distressing experience, stigmation and work-family conflict have not be considered in assessing the intention to work at the infectious treatment centre. 
Table 3: Pre-Service Nurses' Perceptual and Intention to Work at Infectious Centre

\begin{tabular}{lllll}
\hline Variables & Mean & SD & CoV $^{*}$ & Obs. \\
\hline PCSE & 3.230 & 0.220 & 0.068 & 117 \\
PCS & 4.231 & 0.137 & 0.032 & 117 \\
PCWFC & 3.522 & 0.412 & 0.117 & 117 \\
IWITC & 3.789 & 0.093 & 0.025 & 117 \\
\hline
\end{tabular}

*CoV denotes coefficient of variation

Source: Field survey, Yawson (2021)

The findings from the quantitative analyses in Table 3 collaborate with the results from the qualitative data. The interview revealed that the students largely have strong opinion about Ghana handling of the Covid-19 and the news emanating from the media. They generally perceived covid-19 related stress, work-family conflict issues and covid-19 stigma to be very high. It is therefore not surprising that the mean scores of these perceptual factors were very high associated with very low coefficient of variations. The excerpts of the responses are as follows:

One respondent stated:

“.........Even some of us who are not in practice, people look down on us. ............Surprisingly, when cases became intense, some of my own friends did not feel comfortable around me. They think I carry the risk of Covid-19 because I am a student nurse. I could imagine the pains our seniors and those at the treatment centres are going through" PSN \# 7). Another student said:

"At first, I noticed a change in my younger brother's attitude toward me........He was not prompt in accepting items from me. He later disclosed that his friends advised him not to draw close to me else he will be infected with the virus.................................. It took intervention from my Dad to address this challenge" (PSN \# 2)

"My mother worked at one of the treatment centres. Initially, she had to move us to go and stay with my grandmum for fear of infecting us............... She complained about logistical challenges to protect themselves at the treatment centre.................I viewed everyday as nightmare for her" (PSN \# 4)

"My aunt complained about training gap..........she said it is sometimes distressing when you do not know what a covid-19 patient ought to be told about the patient's condition and treatment............. She also said lack of opportunity to talk openly with other units about problems on the Covid-19 unit was distressing " (PSN \# 1)

Another student also emphasised training challenges when he said:

"...................the news from the media make some of us have feel that health practitioners are inadequately prepared to help covid-19 patients and this also increase their own risk exposure............ One friend of mine who is a nurse one said it is very difficult to even get information from physicians regarding the medical condition of covid-19 patients" (PSN \# 9)

Pre-Service Nurses' Perceptual Factors (PCSE, PCS and PCWFC) and Intention to Work at Infectious Treatment Centre in the Future

To determine whether or not the perceptual factors could influence pre-service nurses readiness to work at infectious treatment centres in the future, the study conducted factor analysis on the variables using sphericity and Kaiser-Meyer- Olkin (KMO) tests (Pallant, 2013; Meyers et al., 2013). Prior to that, the study confirmed the internal reliability of the factors using Cronbach's coefficient test with a strict minimum value of 0.6 (Meyers et al., 2013). The KMO test of sample adequacy index value and significant statistics of Bartlett test of sphericity confirmed that the data were appropriate for exploratory factor analysis.

For brevity, some of the details are not reported. However, the study only included factors which have reliability coefficients of not less 0.60 (Meyers et al., 2013). Factors which fall outside this threshold are either deleted entirely or they are combined with other factors especially when they have conceptual fitness. Subsequent to test of multicollinearity where it was found that there was no multicollineraity problem through the correlation matrix, the results from estimating the relationship are reported in Table 4. The estimation controlled for preservice nurses specific demographic factors such as age, gender, marital status and level/forms. 
Table 4 reports the $\mathrm{R}^{2}$ and the f-statistics. It can be observed that $\mathrm{R}^{2}$ for the model is 0.679 . This means that PreService Nurses perceptual factors: Perceived Covid-19 Stressing Experience (PCSE), Perceptual Covid-19 Stigma (PCS), and Perceived Work-Family Conflict of Covid-19 Treatment (PCWFC) are able to explain about $67.9 \%$ of variations in the level of pre-service nurses' Intention to Work at Infectious Treatment Centres (IWITC) in the future. This is relatively high indicating that these perceptual factors are critical in modeling preservice nurses' intentions. Additionally, Table 4 reveals that the f-statistic of the model is 24.072 (0.000). The fstat measures the extent to which the perceptual factors and their control variables are jointly significant in explaining changes in pre-service nurses' intentions to work at infectious treatment centre in the future. Thus, although the individual variables may not be significant, they may be significant when simultaneous employed with other constructs.

It can be observed that all the perceptual factors: Perceived Covid-19 Stressing Experience (PCSE), Perceptual Covid-19 Stigma (PCS), and Perceived Work-Family Conflict of Covid-19 Treatment (PCWFC) have significant negative effect on pre-service nurses' intention to work at infectious treatment centre in the future. Thus, although pre-service nurses could have high positive intention to work at infectious treatment centre as revealed in Table 3, when they continue to have negative perception about these treatment centres, the good intention can be undermined. For the demographic variables: age, marital status and forms/levels of students have negative effect on intention but gender exhibited positive effect. This suggests that female pre-service nurses are more likely to have sustained intention to work at infectious treatment centre. Although insignificant, students' levels or forms have negative effective on intention. Thus, first years are more likely to have positive intention toward working at infectious treatment centre than second and third year students.

Overall the findings support the assertions that mistreatment of healthcare service providers including nurses could make the fight against pandemic and outbreak of infectious diseases fruitless (Bao et al., 2020; Rajkumar,2020; WHO, 2020b).

$\begin{aligned} & \text { Table 4: Relationship between Pre-Service } \\
& \text { Wurses' }\end{aligned}$ Perceptual Factors and Intention to
Work in Infectious Centre in the Future
\begin{tabular}{llllll}
\multicolumn{7}{c}{ Beta } & t-stat & Sig. & $\mathbf{R}^{\mathbf{2}}$ & f-stat \\
\hline Model & & & & 0.679 & $24.072(0.000)$ \\
PCSE & -0.0981 & -2.260 & 0.052 & & \\
PCS & -0.224 & -2.751 & 0.008 & & \\
PCWFC & -0.109 & -1.218 & 0.229 & & \\
Age & -0.0961 & -1.975 & 0.085 & & \\
Gender & 0.2003 & 2.387 & 0.029 & & \\
Marital Status & -0.109 & -1.218 & 0.229 & & \\
Level & -0.160 & -0.987 & 0.472 & & \\
\hline
\end{tabular}

Source: Field survey, Yawson (2021)

\section{Conclusions and Recommendations}

Coronavirus (COVID-19) pandemic exposes healthcare practitioners especially nurses who are often the first point of contact to infection. The perceived experiences and stigmatisation as results of direct exposure to Covid19 Patients, infectious risk and adapting to rigid work schedules could have implications on the intention of preservice nurses in caring for future outbreaks. This has motivated the assessment of pre-service nurses' perceived covid-19 stressing experiences, work-family conflict and stigmatisation in providing healthcare services to covid-19 patients and how these perceptions affect the pre-service nurses' intention toward caring for future outbreaks. Mixed analytical procedure was adopted where both quantitative and qualitative approaches were employed. The study revealed high perception score of stigmatisation, perceived high stressing experience, and high work-family conflict. The study further found that perceptual stigma score, perceived stressing experience and work-family conflict have significant negative influence on the pre-service nurses' intention to provide care for infectious diseases. It is concluded that pre-service nurses' perceived covid-19 stressing experience, perceived Covid-19 stigmatisation and perceived Covid-19 work-family conflict are antecedents of intention to provide care. It is recommended that training of these pre-service nurses should emphasis these experiences and possible stigmas to minimise elements of surprise. Health administrators should regular gather data on nurses' experience and perceive stigmas of epidemics and infectious diseases and integrate them in their training programmes. 


\section{REFERNCE}

Adom, D., Mensah, J. A., \& Osei, M. (2021). The psychological distress and mental health disorders from COVID-19 stigmatization in Ghana. Social sciences \& humanities open, 4(1), 100186.

Andoh, D. (2020, April 15). COVID-19 not a death sentence, stop the stigmatization-Health service boss advises. Retrieved from https://www.graphic.com.ghb.

Braun, V., \& Clarke, V. (2006). Using thematic analysis in psychology. Qualitative research in psychology, 3(2), 77-101.

Berg, B. L. \& Howard, L. (2012). Qualitative Research Methods for the Social Sciences. (8 ${ }^{\text {th }}$ ed). USA: Pearson Educational Inc.

Bu, N.-N., 2020. The 80 thousand COVID-19 survivers are undergoing discriminaton (in Chinese). (access 18 July 2020). http://k.sina.com.cn/article_1690367810_64c0f74201900py8d.html?from=mood

Chen, S. C., Lai, Y. H., \& Tsay, S. L. (2020). Nursing perspectives on the impacts of COVID-19. The Journal of Nursing Research, 28(3), e85 .

Duan W, Bu H, \& Chen Z. (2020) COVID-19-related stigma profiles and risk factors among people who are at high risk of contagion. Soc Sci Med. 2020;266:113425.

Do Duy C, Nong VM, Van AN, Thu TD, Do Thu N, Quang TN. (2020) COVID-19 related stigma and its association with mental health of health-care workers after quarantined in Vietnam. Psychiatry Clin Neurosci. 2020;74(10):566-8.

Earnshaw, V. (2020). Don't let the fear of Covid-19 turn into stigma. Retrieved from Economic and Society website: https://hbr.org/2020/04/dont-let-fear-of-covid-19-turn-into- stigma.

Fraenkel JR, Wallen NE. (2001) How to Design and Evaluate Research in Education. 4th ed. New York: McGraw-Hill, 2000.

Leech, N. L., Gloeckner, G. W., \& Barrett, K. C. (2005). Assumptions of multiple regression : Selecting and Interpreting Inferential Statistics (Chapter 6). (5th edition, pp. 91109).New York: Routledge.

Lu, C., Chen, B., Liu, K., \& Zhu, M. (2020). International cooperation for the coronavirus combat: Results, lessons, and way forward. Shanghai, China: Shanghai Institutes for International Studies.

Ministry of health and family Welfare-India. (2020). Covid-19 India. Ministry of health and family. Welfare website https://www.mohfw.gov.in/.

Molina, A. L. (2018). Strategic Responsiveness and the Minority Public Manager. Public Performance \& Management Review, 41(4), 790-814.

Morens, D. M., \& Fauci, A. S. (2013). Emerging infectious diseases: Threats to human health and global stability. PLoS Pathogens, 9(7), Article e1003467. https://doi.org/ 10.1371/journal.ppat.1003467.

Mostafa, A., Sabry, W., \& Mostafa, N. S. (2020). COVID-19-related stigmatization among a sample of Egyptian healthcare workers. PLoS One, 15(12), e0244172.

Mayo Clinic, 2020. COVID-19 (coronavirus) stigma: what it is and how to reduce it., https://www.mayoclinic.org/diseases-conditions/coronavirus/indepth/coronavirus-stigma/art-20484278 (access 17 Apr 2020).

Pallant, J. F., \& Tennant, A. (2007). An introduction to the Rasch measurement model: an example using the Hospital Anxiety and Depression Scale (HADS). British Journal of Clinical Psychology, 46(1), 1-18.

Rajkumar, R. P. (2020). COVID-19 and mental health: A review of the existing literature. Asian Journal of Psychiatry, 102066. https://doi.org/10.1016/j.ajp.2020.102066.

Taherdoost, H. (2016). Sampling methods in research methodology; how to choose a sampling technique for research. How to Choose a Sampling Technique for Research (April 10, 2016).

Wisdom J \& Creswell J.W (2013). Mixed Methods: Integrating Quantitative and Qualitative Data Collection and Analysis While Studying Patient-Centered Medical Home Models. Rockville, MD: Agency for Healthcare Research and Quality. February 2013. AHRQ Publication No. 13-0028-EF.

World Health Organization, (2020a). Director-General's opening remarks at the media briefing on COVID-19-17 July 2020. [Cited 2020 August 02].Available from: https://www.who.int/dg/speeches/detail/who-director-general-s-opening-remarks-at-the-media-briefingon-covid-19-17-july-2020.

World Health Organization, (2020b). Naming the coronavirus disease (COVID-19) and the virus 
that causes it. https://www.who.int/emergencies/diseases/novel-coronavirus-

2019/technical-guidance/naming-the-coronavirus-disease-(covid-2019)-and-the-virusthat-causes-it (access 11 Feb 2020).

Xiao, C. (2020). A novel approach of consultation on 2019 novel coronavirus (COVID-

19). Related psychological and mental problems: Structured letter therapy.

Psychiatr.Invest., 17(2), 175-176.

Yanow, D., \& Schwartz-Shea, P. (2014). Interpretation and method: Empirical research methods and the interpretive turn, (2nd ed.). London \& New York: M. E. Sharpe \& Routledge.

Yuan, Yan-Jie , Qing-E, Ling, Teris, Todd, Guo-Qing and Yu-Tao (2020) COVID-19-related stigma and its socio demographic correlates: a comparative study; Globalization and Health (2021) 17:54 https://doi.org/10.1186/s12992-021-00705-4 